\title{
Analysis of Chloramphenicol and Its Related Compound 2-Amino-1-(4-nitrophenyl)propane-1,3-diol by Reversed-Phase High-Performance Liquid Chromatography with UV Detection
}

\author{
Fuad Al-Rimawi ${ }^{1}$ and Maher Kharoaf ${ }^{2}$ \\ ${ }^{1}$ Faculty of Science and Technology, Al-Quds University, P.O. Box 20002, East Jerusalem, Palestine \\ ${ }^{2}$ Research and Development Department, Jerusalem Pharmaceutical Co., P.O. Box 3570, Al-Bireh, Palestine \\ Correspondence should be addressed to Fuad Al-Rimawi, fuad_12345@yahoo.com
}

Received 18 May 2011; Revised 9 July 2011; Accepted 10 July 2011

Academic Editor: Wenkui Li

Copyright (c) 2011 F. Al-Rimawi and M. Kharoaf. This is an open access article distributed under the Creative Commons Attribution License, which permits unrestricted use, distribution, and reproduction in any medium, provided the original work is properly cited.

\begin{abstract}
A simple and stability-indicating liquid chromatographic method is developed for the analysis of chloramphenicol and its related compound 2-amino-1-(4-nitrophenyl)propane-1,3-diol in two pharmaceutical forms. Liquid chromatography with a UV detector at a wavelength of $278 \mathrm{~nm}$ using a reversed phase C18 stationary phase has been employed in this study. Isocratic elution is employed using a mixture of sodium pentanesulfonate solution $(0.012 \mathrm{M})$, acetonitrile, and glacial acetic acid $(85: 15: 1$, v/v). This new method is validated in accordance with USP requirements for new methods for assay determination, which include accuracy, precision, specificity, linearity and range. This method shows enough selectivity, sensitivity, accuracy, precision, and linearity range to satisfy Federal Drug Administration/International Conference on Harmonization regulatory requirements. The current method demonstrates good linearity over the range of $0.04-0.16 \mathrm{mg} / \mathrm{mL}$ of chloramphenicol. The accuracy of the method is $100.0 \%$ with a relative standard deviation of $0.1 \%$. The precision of this method reflected by relative standard deviation of replicates is $0.1 \%$. The method is sensitive with a detection limit of $0.005 \%$ for chloramphenicol. The related substance of chloramphenicol (2-amino-1(4-nitrophenyl)propane-1,3-diol) can be selectively determined with a good resolution in two pharmaceutical forms: eye ointment and eye drops.
\end{abstract}

\section{Introduction}

Chloramphenicol is a bacteriostatic antimicrobial compound originally derived from the bacterium Streptomyces venezuelae, isolated by David Gottlieb, and introduced into clinical practice in 1949, its structure is shown in Figure 1. It was the first antibiotic to be manufactured synthetically on a large scale. Chloramphenicol is effective against a wide variety of microorganisms. Chloramphenicol is used in eye drops or ointment for bacterial conjunctivitis for the treatment of bacterial conjunctivitis [1].

Chloramphenicol loses its activity by the hydrolysis of the amide group to give 2-amino-1-(4-nitrophenyl)propane1,3-diol, its structure is shown in Figure 1. This degradation product (hydrolysis product) of chloramphenicol commonly occurs in pharmaceutical preparations [2]. Chloramphenicol has also many related compounds and impurities which may present in the pharmaceutical drug. As impurities in pharmaceutical drugs can change the effects and side effects of a drug significantly, it is important to have an accurate method for determination of these impurities. In this sense, a selective, stability-indicating method is needed for the analysis of chloramphenicol from its degradation products, related compounds, and impurities, for example, 2-amino1-(4-nitrophenyl)propane-1,3-diol.

Many methods have been employed for the analysis of chloramphenicol in the presence of its impurities, intermediates, and degradation products. Vigh and Inczédy have reported in 1976 an HPLC method for the separation of six chloramphenicol intermediates which formed during its preparation [1]. A method for the determination of chloramphenicol and its monosuccinate ester in pig plasma 
<smiles>O=C(N[C@H](CO)[C@H](O)c1ccc([N+](=O)[O-])cc1)C(Cl)Cl</smiles>

(a)<smiles>N[C@@H](CO)[C@H](O)c1ccc([N+](=O)[O-])cc1</smiles>

(b)

Figure 1: Structure of chloramphenicol (I) and its potential impurity 2-amino-1-(4-nitrophenyl)propane-1,3-diol (II).

has also been described by Wiese et al. using RP-18 column and phosphate buffer $\mathrm{pH} 4.9$ containing 30\% methanol as mobile phase [3]. Boer and Pijnenburg have developed an HPLC method for the simultaneous determination of chloramphenicol and its most important degradation products. C18-reversed phase column has been employed with a mobile phase consisting of boric acid solution and acetonitrile $(60: 40, \mathrm{v} / \mathrm{v})$ adjusted to $\mathrm{pH}$ of 3.0 [4]. Seth and Bannerjee has also determined chloramphenicol in the presence of its degradation products in drug formulations by using polarographic method [5]. In 1991, Sudana and Chogare have employed an RP-HPLC method for the separation of chloramphenicol and benzocaine from their impurities and excipients present in topical solutions and suppositories [6].

In 2001, an HPLC method was described for the analysis of chloramphenicol and its impurities resulted from gamma irradiation of chloramphenicol in eye ointment [7]. Chloramphenicol and its glucuronide in some food products (chicken meat, seafood, and honey) have also been determined in 2004 by Bogusz et al. by reverse-phase liquid chromatography-electrospray negative ionization mass spectrometry using acetonitrile and ammonium formate $10 \mathrm{mM}, \mathrm{pH} 3.0(40: 60, \mathrm{v} / \mathrm{v})$ as mobile phase at flow rate of $0.3 \mathrm{~mL} / \mathrm{min}$ [8]. In 2006, Visky et al. have described an HPLC method for the separation of seven different drug substances from their respective impurities, among these drug substances is chloramphenicol and its impurities [9].

Lebelle et al. have reported an RP-HPLC method for determination of Chloramphenicol and 2-amino-1(4-nitrophenyl)propane-1,3-diol [2]. Ali has described an HPLC method for the separation of chloramphenicol from this impurity in a number of pharmaceutical preparations [10]. However, this method is not validated according to USP and ICH requirements for new method for assay determination. The USP describes a reversed-phase HPLC method for the determination of chloramphenicol in raw material as well as in many pharmaceutical dosage forms [11]. However, this method is used for the analysis of chloramphenicol without this degradation product (2-amino-1(4-nitrophenyl)propane-1,3-diol). Furthermore, the British Pharmacopoeia (BP) method for the determination of chloramphenicol (assay) in raw material as well as in many pharmaceutical forms is UV spectrophotometry or colorimetry [12] which cannot detect this impurity. Therefore, a stabilityindicating test method is needed for the determination of chloramphenicol in the presence of this impurity. The objective of the current paper, therefore, is to develop and validate a method for the determination of chloramphenicol with this impurity in two pharmaceutical dosage forms: eye ointment and eye drops, by using a reversed-phase HPLC method. Validation of the method will be performed according to the requirements of USP for assay determination which include accuracy, precision, specificity, linearity and range. Additionally, in order to meet the regulatory guidance of the Federal Drug Administration $\backslash$ International Conference on Harmonization (ICH) [13], chloramphenicol will be forcibly degraded in acidic, basic, and strong oxidizing agent solutions. The method is able to detect and quantitatively estimate the degradation products generated.

\section{Experimental}

2.1. Chemicals. Acetonitrile HPLC grade is from J. T. Baker (NJ, USA). Glacial acetic acid, sodium pentanesulfonate, hydrochloric acid, sodium hydroxide, and hydrogen peroxide are from Merck (Darmstadt, Germany). Chloramphenicol RS, 2-amino-1-(4-nitrophenyl)propane-1,3-diol RS are from USP (Rockville, Md, USA).

2.2. Apparatus. HPLC system (Merck Hitachi LaChrom Elite HPLC system, Japan) with an L-2130 pump, an L2200 autosampler, L-2300 column oven, and L-2490 UV detector was employed. The Ezchrom Elite software was employed. The chromatographic analysis was performed on HX749288, LiChroCart, HPLC-cartage Purospher STAR RP-18 endcapped $(5 \mu \mathrm{m})$, (150 mm length, $4.6 \mathrm{~mm}$ inner diameter) (Waters Corporation, Milford, Mass, USA). The column is kept at room temperature.

2.3. Standard Solutions and HPLC Conditions. Sodium pentanesulfonate solution was prepared by dissolving $2.1 \mathrm{~g}$ in $1000 \mathrm{~mL}$ of water. A filtered and degassed mixture of sodium pentanesulfonate solution, acetonitrile, and glacial acetic acid (different volume fractions) has been tested as a mobile phase for the separation of chloramphenicol and 2-amino-1-(4-nitrophenyl)propane-1,3-diol. The best combination was 85:15:1 of sodium pentanesulfonate solution : acetonitrile : glacial acetic acid. Different flow rates $(1.0,1.5$, and $2.0 \mathrm{~mL} / \mathrm{min})$ have been tested. UV detection was performed at $278 \mathrm{~nm}$, and injection volume was $10 \mu \mathrm{L}$.

Stock standard solution of chloramphenicol was prepared by dissolving $100 \mathrm{mg}$ of chloramphenicol in $100 \mathrm{~mL}$ 
of water to obtain a solution having a known concentration of $1.0 \mathrm{mg}$ per $\mathrm{mL}$ chloramphenicol.

Nominal standard solution was prepared by diluting $5 \mathrm{~mL}$ of stock standard solution to $50 \mathrm{~mL}$ mobile phase to obtain a solution having a known concentration of $0.10 \mathrm{mg}$ per mL chloramphenicol.

Standard solution of 2-amino-1-(4-nitrophenyl)propane-1,3-diol was prepared by dissolving $10 \mathrm{mg}$ in $100 \mathrm{~mL}$ of methanol, and diluting $25 \mathrm{~mL}$ of this solution to $50 \mathrm{~mL}$ mobile phase to obtain a solution having a known concentration of $0.05 \mathrm{mg}$ per $\mathrm{mL}$ 2-amino-1-(4-nitrophenyl)propane1,3-diol.

Nominal solutions of the formulated chloramphenicol eye drops were prepared by diluting a volume of the product equivalent to $50 \mathrm{mg}$ of chloramphenicol $(10 \mathrm{~mL}$ of the product) to $50 \mathrm{~mL}$ with water, then diluting $5.0 \mathrm{~mL}$ of the resulting solution to $50 \mathrm{~mL}$ with water.

Chloramphenicol in eye ointment was extracted as follows: $0.4 \mathrm{~g}$ of the ointment was dissolved in $50 \mathrm{~mL}$ of $\mathrm{n}$ hexane, then extracted with $50 \mathrm{~mL}$ of water (three times). The extracts were combined in a 200-ml volumetric flask, and diluted to volume with water.

\section{Results and Discussion}

3.1. Method Development. A reversed-phase C18, $5 \mu \mathrm{m}$, $150 \mathrm{~mm}$ length, and $4.6 \mathrm{~mm}$ inner diameter column maintained at ambient temperature was used for the separation of chloramphenicol and 2-amino-1-(4-nitrophenyl)propane1,3-diol.

Regarding the mobile phase, a mixture of sodium pentanesulfonate solution, acetonitrile, and glacial acetic acid was used. In order to improve the separation and peak symmetry, the mobile phase composition was varied until optimum composition was selected $(85: 15: 1, \mathrm{v} / \mathrm{v})$. Isocratic elution at flow rate of $2.0 \mathrm{~mL} / \mathrm{min}$ has been employed in this study. Wavelength of 278 was selected to be used for UV detection. The column is kept at room temperature during this study.

After this optimization, this method has been used for the separation of chloramphenicol from 2-amino-1-(4nitrophenyl)propane-1,3-diol (Figure 2). A good separation with adequate resolution has been obtained.

3.2. Method Validation. After method development, the validation of the current test method for chloramphenicol has been performed in accordance with USP requirements for assay determination (Category I: analytical methods for quantitation of active ingredients in finished pharmaceutical products) which include accuracy, precision, specificity, linearity, and range [13].

3.2.1. Linearity and Range. Linearity is the ability of a method to elicit test results that are directly proportional to analyte concentration within a given range. Range is the interval between the upper and lower levels of analyte that have been demonstrated to be determined with precision, accuracy, and linearity using the method as written.

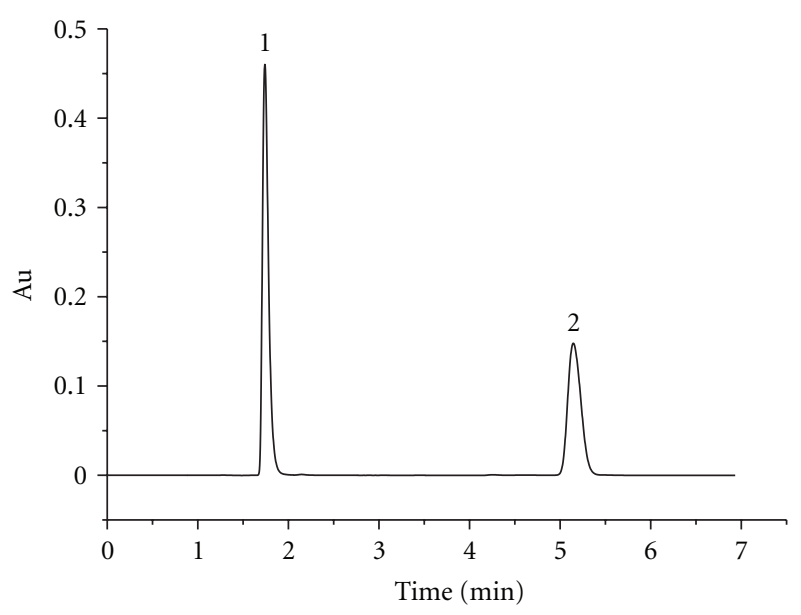

FIGURE 2: Chromatogram of 2-amino-1-(4-nitrophenyl)propane1,3-diol (1) and chloramphenicol (2), $0.05 \mathrm{mg} / \mathrm{mL}$ of each. Mobile phase: sodium pentanesulfonate solution, acetonitrile, glacial acetic acid $(85: 15: 1, \mathrm{v} / \mathrm{v})$, flow rate $2.0 \mathrm{~mL} / \mathrm{min}$, injection volume $10 \mu \mathrm{L}$. Column: reversed phase C18, $5 \mu \mathrm{m}, 15 \mathrm{~cm}$ length, $4.6 \mathrm{~mm}$ inner diameter, UV detection: $278 \mathrm{~nm}$. Peak asymmetry and theoretical plates of 2-amino-1-(4-nitrophenyl)propane-1,3-diol are 1.36, and 3287 , respectively. Peak asymmetry, theoretical plates, and resolution of chloramphenicol are $1.25,5781$, and 11.3 , respectively.

A minimum of five concentration levels, along with certain minimum specified ranges are required [14]. Acceptance criteria for linearity are that the correlation coefficient $\left(R^{2}\right)$ is not less than 0.990 for the least squares method of analysis of the line.

Standard solutions covering the range between 40 and $160 \%$ of the nominal standard concentration $(0.1 \mathrm{mg} / \mathrm{mL}$ chloramphenicol) have been prepared by diluting specific volume of the stock standard to get several concentrations $(0.04,0.05,0.08,0.1,0.12$, and $0.16 \mathrm{mg} / \mathrm{mL})$. Then, these standards have been chromatographed using UV detector at $278 \mathrm{~nm}$. Three runs have been performed for every concentration. The peak responses, for example, peak area have been recorded and plotted versus standard concentrations. Results have shown that the method is linear over the specified range with $R^{2}$ of 0.9996 , insignificant $y$-intercept $(-11434)$, and a slope of $3 \times 10^{07}$ has also been obtained. These findings demonstrate linearity of this method over the specified range.

3.2.2. Accuracy. The accuracy of an analytical procedure measures the closeness of agreement between the value, which is accepted either as a conventional true value or an accepted reference value and value found. Accuracy is measured as the percent of analyte recovered by assay, by spiking samples in a blind study [15].

To document accuracy, a minimum of nine determinations over a minimum of three concentration levels covering the specified range (e.g., three concentrations, three replicates for each) were collected. It is performed at 80,100 , and $120 \%$ levels of label claim. At each level studied, replicate samples are evaluated. The RSD of the replicates provides 
TABLE 1: Accuracy (\% recovery) of chloramphenicol in eye ointment and eye drops formulations at three concentration levels.

\begin{tabular}{|c|c|c|c|c|}
\hline \multirow{2}{*}{ Chloramphenicol concentration (mg/L) } & \multicolumn{2}{|c|}{ \% Acuracy (recovery) } & \multicolumn{2}{|c|}{ RSD for 3 replicates } \\
\hline & Eye ointment & Eye drops & Eye ointment & Eye drops \\
\hline 0.08 & 100.6 & 99.5 & $0.3 \%$ & $0.3 \%$ \\
\hline 0.1 & 100.0 & 100.0 & $0.3 \%$ & $0.05 \%$ \\
\hline 0.12 & 101.2 & 99.1 & $0.4 \%$ & $0.2 \%$ \\
\hline
\end{tabular}

the analysis variation and gives an indication of the precision of the test method. Moreover, the mean of the replicates, expressed as \% of label claim, indicates the accuracy of the test method. The mean recovery of the assay should be within $100 \pm 2.0 \%$ at each concentration over the range of $80-120 \%$ of nominal concentration [15].

To prepare accuracy standard solutions, placebo of the drug formulation, for example, eye drops or eye ointment has to be prepared according to the formulation procedure. To the required quantity of placebo, a known quantity of chloramphenicol with the same proportion as in the drug formulation has been added to get three concentrations (0.08, 0.1 (nominal concentration), and $0.12 \mathrm{mg} / \mathrm{mL}$ of chloramphenicol). These standards, then, have been chromatographed. Three runs have been performed for every concentration, and then peak area has been recorded. The average recovery and the RSD for each level have been calculated. Results have shown that the mean recovery of the assay for both drug products (eye drops and eye ointment) is within $100 \pm 2.0 \%$ at each concentration, and the RSD is lower than $1.0 \%$, see Table 1 . Furthermore, results have shown that recovery data obtained was within the 99.8$101.53 \%$ range for eye ointment formulation (mean = $100.6 \%$ ) and $99.0-100.0 \%$ range for eye drops (mean = 99.5\%), see Table 1 .

3.2.3. Precision. Precision is the measure of the degree of repeatability of an analytical method under normal operation and is normally expressed as the RSD for a statistically significant number of samples. It is determined from a minimum of nine determinations covering the specified range of the procedure (e.g., three levels, three repetitions each), or from a minimum of six determinations, at $100 \%$ of the test or target concentration. RSD for replicate injections should not be greater than $1.5 \%$ [16].

The RSD of the peak areas for the recovery data analyzed in accuracy study (see Section 3.2.2) for each level (80\%, $100 \%$, and $120 \%$ of the nominal concentration) has been calculated, and it has been found to be less than $1.0 \%$ for each level, see Table 2. The RSD of the peak areas of six replicate injections for the nominal standard concentration (100\%) has also been calculated to be $0.1 \%$. These results show that the current method for chloramphenicol analysis is repeatable.

3.2.4. Specificity (Stability Indicating Evaluation). Specificity is the ability to assess unequivocally the analyte in the presence of components that may be expected to be present, such as impurities, degradation products, and matrix components
TABLE 2: Precision of chloramphenicol analysis in eye ointment and eye drop formulations at three concentration levels.

\begin{tabular}{lcc}
\hline $\begin{array}{l}\text { Chloramphenicol concentration } \\
(\mathrm{mg} / \mathrm{L})\end{array}$ & Rye ointment & Eye drops \\
\hline 0.08 & $0.4 \%$ & $0.8 \%$ \\
0.1 & $0.7 \%$ & $0.2 \%$ \\
0.12 & $0.4 \%$ & $0.6 \%$ \\
\hline
\end{tabular}

[17]. It is a measure of the degree of interferences from such components, ensuring that a peak response is due to a single component only. Specificity is measured and documented in a separation by the resolution, plate count (efficiency), and tailing factor. Resolution between the active ingredient, for example, chloramphenicol and all the components have to be at least 1.5. Efficiency of the column is not less than 1500 theoretical plates for chloramphenicol peak, and the tailing factor is not more than 1.5 .

Specificity may be demonstrated by enhancing degradation of the chloramphenicol under stress conditions (acid and base hydrolysis, and oxidation). Accordingly, $10 \mathrm{~mL}$ of $2 \mathrm{M}$ hydrochloric acid, $10 \mathrm{~mL}$ of $2 \mathrm{M}$ sodium hydroxide, and $10 \mathrm{~mL}$ of $35 \%$ hydrogen peroxide have been added to $100 \mathrm{~mL}$ of assay solution. These solutions have been chromatographed, and all the peaks in the chromatograms are recorded. Results showed that the resolution between chloramphenicol and all other degradation products or components is higher than 1.5 which indicates that the method is specific for determination of chloramphenicol and can separate and detect possible degradation products, see Figure 3.

We have also found that chloramphenicol is stable in both acidic solution and hydrogen peroxide solution (it gives no degradation product when it is exposed to hydrochloric acid or hydrogen peroxide solutions). On the other hand, chloramphenicol has been degraded slightly in basic solution to give 2-amino-1-(4-nitrophenyl)propane-1,3-diol, see Figure 3. Its degradation has been further enhanced by heating sodium hydroxide solution at $60^{\circ} \mathrm{C}$ for 60 minutes to give $100 \%$ of 2-amino-1-(4-nitrophenyl)propane-1,3-diol.

3.2.5. Detection Limit and Quantitation Limit. Detection limit is the lowest concentration of analyte in a sample that can be detected, but not necessarily quantitated, under the stated experimental conditions [16]. Detection limit can be determined by preparing a solution that is expected to produce a response that is approximately 3 to 10 times base line noise. The solution is injected three times, and the signal 


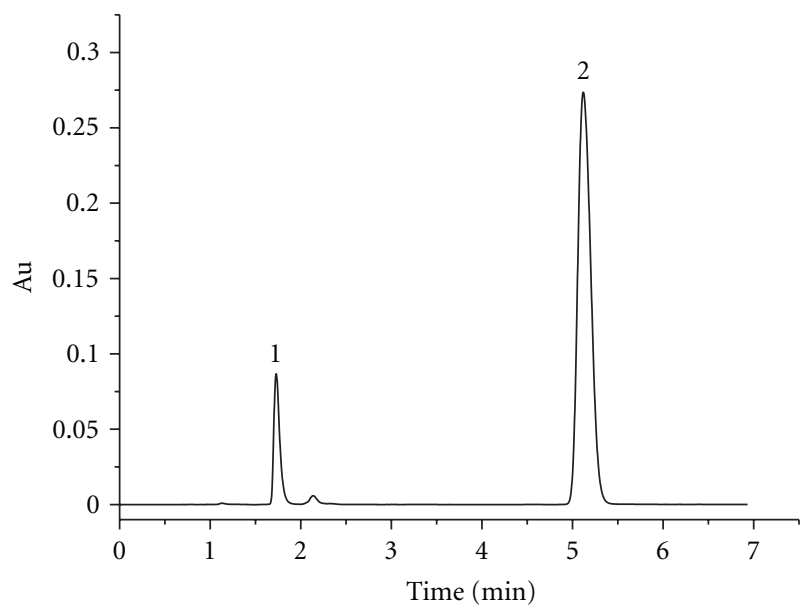

Figure 3: Chromatogram of chloramphenicol, $0.1 \mathrm{mg} / \mathrm{mL}$ of cloramphenicol, added to it $10 \%$ of $2 \mathrm{M}$ sodium hydroxide. Analytes, (1): 2-amino-1-(4-nitrophenyl)propane-1,3-diol, (2): chloramphenicol. Other experimental conditions are the same as in Figure 2. Peak asymmetry and theoretical plates of 2-amino-1-(4nitrophenyl)propane-1,3-diol are 1.41, and 3461, respectively. Peak asymmetry, theoretical plates, and resolution of chloramphenicol are $1.24,5831$, and 10.6 , respectively.

and the noise for each injection are recorded. Each signal to noise ratio $(\mathrm{S} / \mathrm{N})$ is then calculated, and averaged. The concentration of the solution is used for determination of the detection limit if the average $\mathrm{S} / \mathrm{N}$ ratio is between 3 and 10 . If it is not between 3 and 10 , the solution concentration is modified as necessary and the experiment is repeated. The limit of detection (LOD) may be expressed as

$$
\mathrm{LOD}=3.3 \frac{\sigma}{S}
$$

where $\sigma$ is the standard deviation of the response, and $S$ is the slope of the calibration curve.

Limit of quantitation can be determined in the same manner but using the formula $10 \sigma / \mathrm{S}$.

Results showed that detection limit and quantitation limit for chloramphenicol using this method are $0.005 \%$ and $0.015 \%$, respectively.

After successful development and validation of this method, we have employed it for the analysis of chloramphenicol in two drug formulations (eye ointment and eye drops), see Figure 4.

\section{Conclusion}

A simple, accurate, and precise stability-indicating HPLC analytical method has been developed and validated for the routine analysis of chloramphenicol and 2-amino-1-(4nitrophenyl)propane-1,3-diol in two pharmaceutical dosage forms. The results of stress testing undertaken according to the International Conference on Harmonization guidelines reveal that the method is selective and stability-indicating.

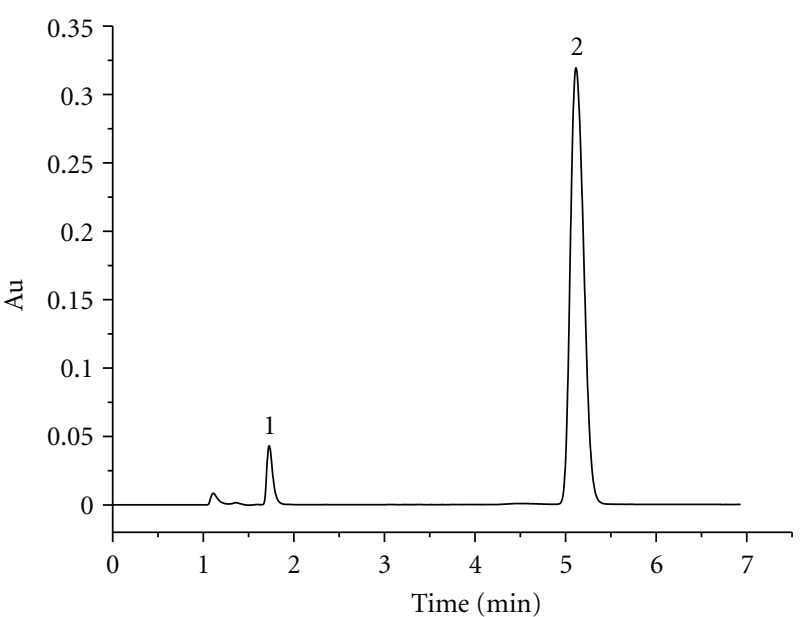

Figure 4: Chromatogram of chloramphenicol in eye drops $(0.1 \mathrm{mg} / \mathrm{mL}$ of chloramphenicol). Analytes, (1): 2-amino-1-(4nitrophenyl)propane-1,3-diol, (2): chloramphenicol. Other experimental conditions are the same as in Figure 2. Peak asymmetry and theoretical plates of 2-amino-1-(4-nitrophenyl)propane-1,3diol are 1.38 and 3373, respectively. Peak asymmetry, theoretical plates, and resolution of chloramphenicol are 1.24, 5514, and 10.2, respectively.

The proposed method has the ability to separate chloramphenicol from its related compounds, impurities, degradation products, and excipients found in the pharmaceutical dosage forms.

\section{Acknowledgment}

The authors would like to thank gratefully Jerusalem Pharmaceuticals for its support and providing them with the necessary instruments/apparatus to perform this study.

\section{References}

[1] G. Vigh and J. Inczédy, "Separation of some chloramphenicol intermediates by high-pressure liquid chromatography," Journal of Chromatography, vol. 116, no. 2, pp. 472-474, 1976.

[2] M. J. LeBelle, D. C. Young, K. C. Graham, and W. L. Wilson, "High-performance liquid chromatographic determination of chloramphenicol and 1-(4'-nitrophenyl)-2-aminopropane1,3-diol in pharmaceutical formulations," Journal of Chromatography, vol. 170, no. 1, pp. 282-287, 1979.

[3] B. Wiese, K. Martin, and J. Hermansson, "Determination of chloramphenicol and its monosuccinate ester in piglet plasma using HPLC," Chromatographia, vol. 15, no. 12, pp. 737-742.

[4] Y. Boer and A. Pijnenburg, "HPLC determination of chloramphenicol degradation in eye drops," Pharmacy World \& Science, vol. 5, no. 3, pp. 95-101, 1983.

[5] S. Seth and N. R. Bannerjee, "Estimation of chloramphenicol in presence of its degradation products," Indian Journal of Pharmaceutical Sciences, vol. 49, no. 2, pp. 58-60, 1987.

[6] G. S. Sadana and A. B. Ghogare, "Simultaneous determination of chloramphenicol and benzocaine in topical formulations by high-performance liquid chromatography," Journal of 
Chromatography, vol. 542, no. 2, pp. 515-520, 1991.

[7] L. Hong and H. Altorfer, "Determination of assay and impurities of gamma irradiated chloramphenicol in eye ointment," Journal of Pharmaceutical and Biomedical Analysis, vol. 24, no. 4, pp. 667-674, 2001.

[8] M. J. Bogusz, H. Hassan, E. Al-Enazi, Z. Ibrahim, and M. Al-Tufail, "Rapid determination of chloramphenicol and its glucuronide in food products by liquid chromatographyelectrospray negative ionization tandem mass spectrometry," Journal of Chromatogr B, vol. 807, no. 2, pp. 343-356, 2004.

[9] D. Visky, E. Haghedooren, P. Dehouck et al., "Facilitated column selection in pharmaceutical analyses using a simple column classification system," Journal of Chromatography A, vol. 1101, no. 1-2, pp. 103-114, 2006.

[10] S. L. Ali, "Separation and determination of the hydrolysis products of chloramphenicol in pharmaceutical preparations by high-performance liquid chromatography," Journal of Chromatography, vol. 154, no. 1, pp. 103-105, 1978.

[11] United States Pharmacopoeia-National Formulary 26, vol. 31, Rockville, MD, USA, 2008.

[12] British Pharmacopoeia Commission, British Pharmacopoeia, London, UK, 2007.

[13] "Stability testing of new active substances and medicinal products," ICH Topic Q1A. ICH Harmonized Tripartite Guidelines, ICH, Surrey, UK, CPMP/ICH/280/95, 1996.

[14] United States Pharmacopoeia, National Formulary, Validation of Compendial Methods, chapter 1225, United States Pharmacopoeia, Rockville, Md, USA, 2007.

[15] "Validation of analytical procedures-PA/PH/OMCL (05) 47 DEF," in Proceedings of the International Conference on Harmonization (ICH), OMCL Network/EDQM of the Council of Europe, June 2005.

[16] J. Mark Green, "A practical guide to analytical method validation," Analytical Chemistry, pp. 305A-309A, 1996.

[17] L. Huber, "Validation of analytical methods," in Validation and Qualification in the Analytical Laboratories, L. Huber, Ed., p. 107, Interpharm Press, Buffalo Grove. Ill, USA, 1998. 


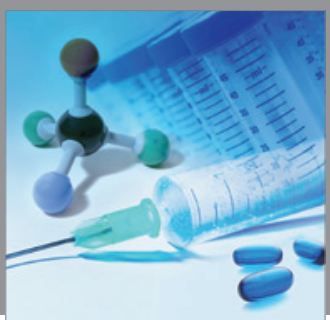

International Journal of

Medicinal Chemistry

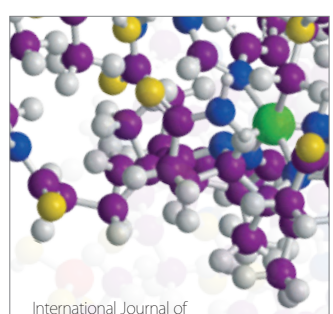

Carbohydrate Chemistry

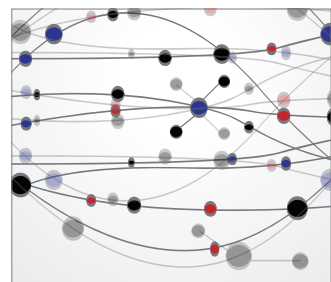

The Scientific World Journal
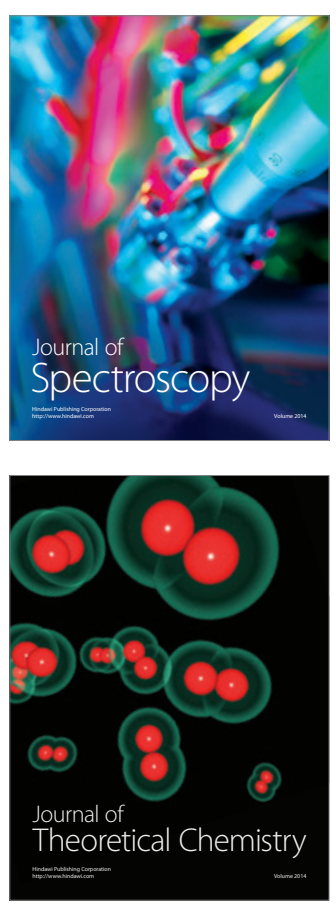
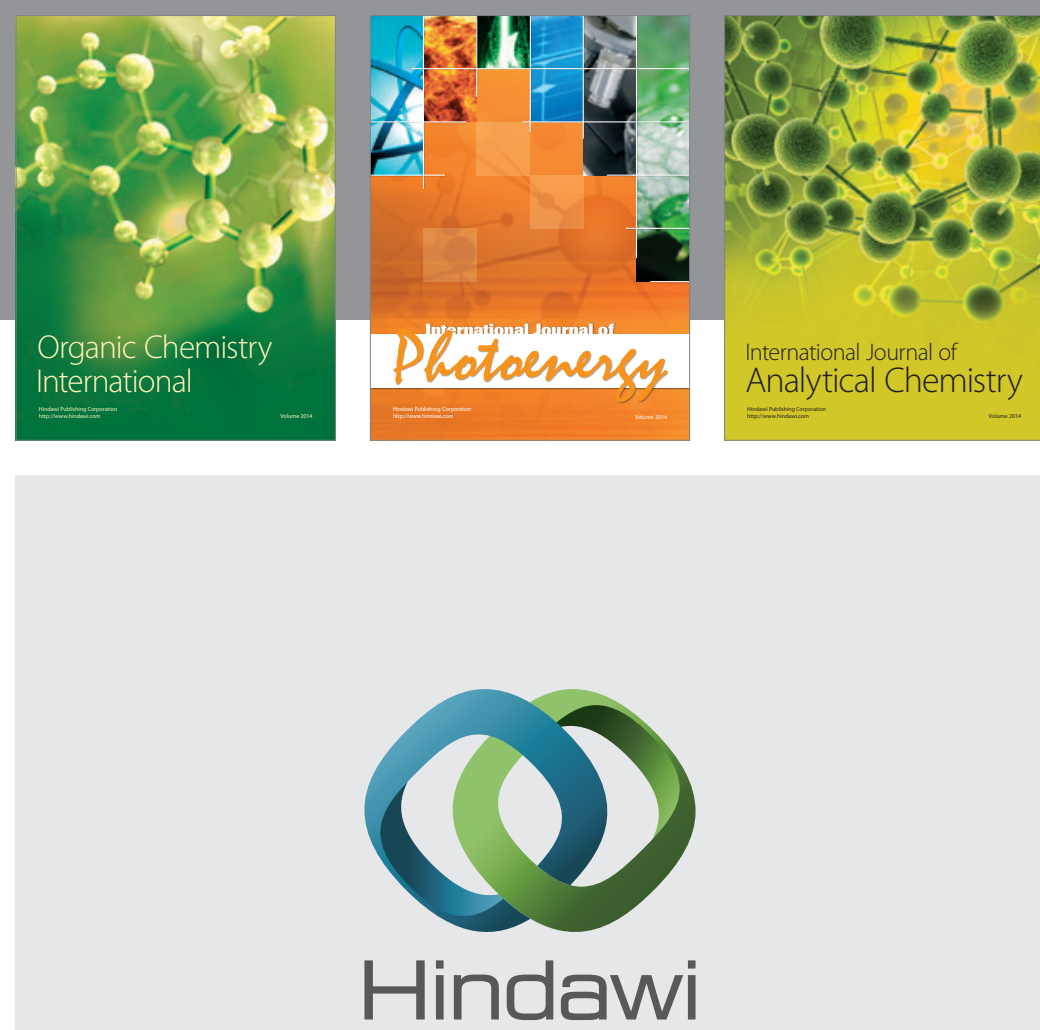

Submit your manuscripts at

http://www.hindawi.com
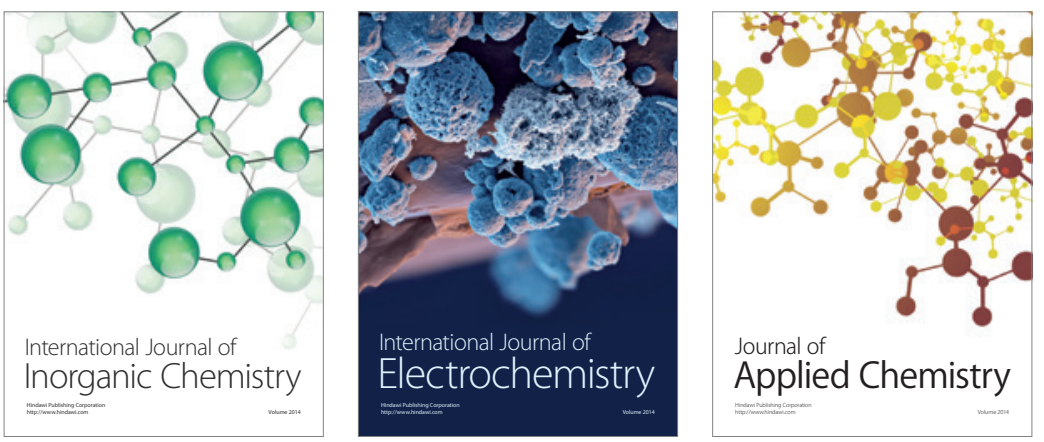

Journal of

Applied Chemistry
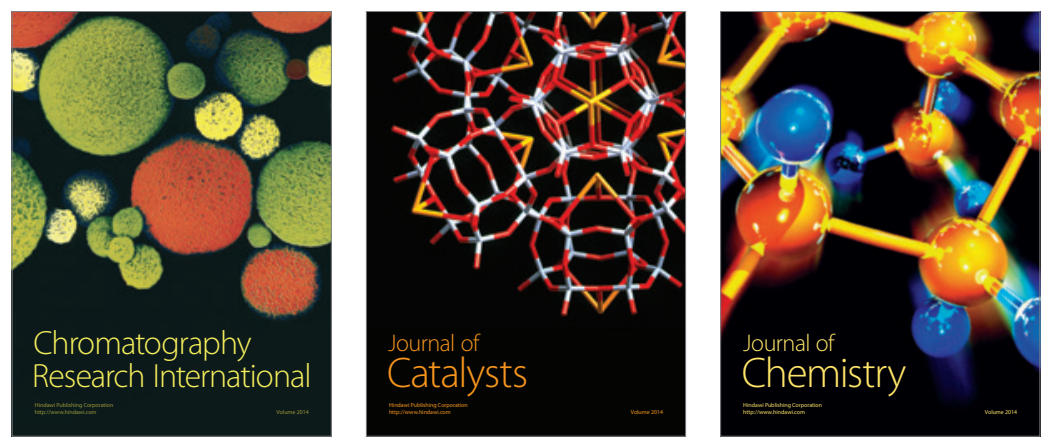
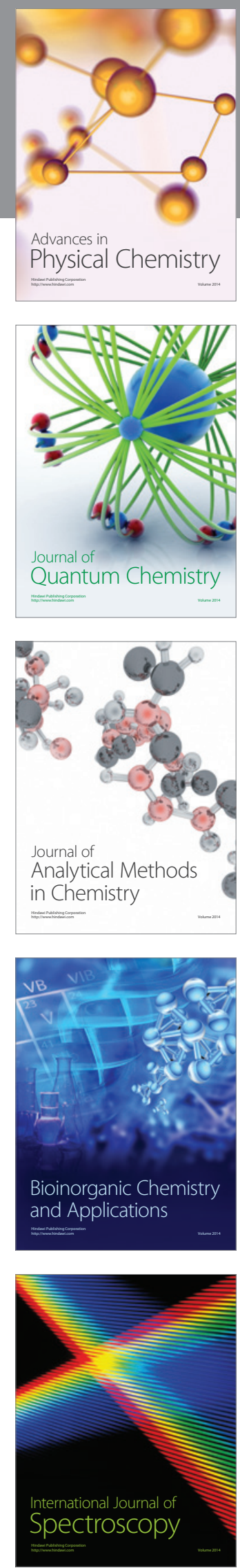